\title{
ESCENARIOS MUNDIALES Y REGIONALES DE LA EDUCACIÓN SUPERIOR
}

\author{
Francisco LóPEZ SEgRera ${ }^{1}$
}

\begin{abstract}
1 Asesor Académico de la Global University Network for Innovation (GUNI). Miembro del Foro UNESCO de educación superior, investigación y conocimiento. Miembro del Grupo de trabajo de CLACSO sobre Universidad y Sociedad. Presidente de la Red Latinoamericana de Estudios Prospectivos. Director del IESALC, UNESCO (1999-2001). Investigador Titular Adjunto del Centro Juan Marinello (Cuba). Profesor Titular Adjunto del Instituto Superior de Relaciones Internacionales (Cuba).
\end{abstract}

Resumen: El presente artículo presenta un análisis y un ejercicio prospectivo de posibles escenarios y alternativas globales de la educación superior, de entre ellos: modelo tradicional; modelo de libre mercado; educación permanente; red global de instituciones; desaparición de las universidades. Estos escenarios tienen relaciones con el desarrollo de temas de largo contexto, como la globaliazación, la sociedad del conocimiento, las redes de cooperación regionales, la educación permanente y sobre todo los desdoblamientos de la disputa respecto al significado de la educación superior como bien público o comercio rentable.

Palabras-clave: Educación Superior; Escenarios globales; Modelos de Universidad; Organismos multilaterales; Privatización; Transnacionalización.

Global and Regional Scenarios of Higher Education

Abstract: The article presents an analysis and a prospective exercise of possible global scenarios and alternatives for higher education, such as: traditional model; free market model; permanent education; global chain of institutions; disappearance of the universities. These scenarios are related to the development of the large context topics, such as globalization, the society of knowledge, the chains of regional cooperation, permanent education and all the unfoldings of the dispute on the meaning of higher education as a public good or a profitable business.

Key-words: Higher Education, Global settings, University models, Multilateral organisms, Privatization, Transnationalization. 


\section{Escenarios y Alternativas Globales}

La universidad medieval - Bolonia, Paris, Salamanca - dio paso en los siglos siguientes a modelos cada vez más rígidos que se articularon en torno a tres enfoques: el modelo inglés, o sistema universitario residencial de Oxford; el modelo francés, basado en las "grandes escuelas" o facultades, denominado "sistema napoleónico", en que la universidad, sometida a la tutela y guía del estado, tiene como función formar profesionales; y el modelo alemán de investigación, derivado de la universidad de Humboldt. Estos modelos predominaron durante siglo y medio. A mediados del siglo $\mathrm{XX}$ emergieron modelos mixtos, como el modelo norteamericano que hacía hincapié en la estructura departamental, hoy en discusión por su fragmentación excesiva. La universidad actual, no solo en los países desarrollados de la OECD, sino también en los países en desarrollo de Asia, África, los Estados Árabes y de América Latina y el Caribe, se asemeja a alguno de esos modelos o bien a una combinación de los mismos. En el caso de los países en desarrollo, estos modelos llegaron junto con la dominación colonial y neocolonial. Pese a las características endógenas de los sistemas de educación superior de los países en desarrollo y de sus peculiaridades regionales y nacionales, son estos modelos los que prevalecen. También las políticas globales de UNESCO y del Banco Mundial, han contribuido a esta tendencia homogeneizadora. De ahí que los cambios en los sistemas de educación superior de las zonas metropolitanas del centro (OECD) repercutan con fuerza en la periferia. (SEGRERA LÓPEZ, 2006).

Los Escenarios que presentaremos a continuación fueron formulados teniendo en mente a los países de la OECD, pero sin duda tienen un espectro más amplio por lo señalado en el párrafo anterior ${ }^{2}$. En un eje del gráfico 1 (educación terciaria inicial) se considera que el $80 \%$ de los futuros estudiantes universitarios - al igual que hoy - tendrán menos de 23 años. En el otro extremo (educación permanente, lifelong learning) se refiere a una situación en que los adultos asistirán a la universidad en diferentes períodos de sus vidas, $50 \%$ de los estudiantes universitarios tendrán más de 40 años. (Lancrin, 2004; Ver Gráfico 1)

2 Nos hemos basado en la excelente formulación de escenarios de Lancrin (2004) para - a la vez que los resumimos - incorporar a ellos nuestras reflexiones. 
Tabla 1. Seis Escenarios para el futuro de las Universidades.

\begin{tabular}{|c|c|c|c|c|c|c|c|}
\hline & & \multicolumn{6}{|c|}{ Scenario: } \\
\hline & & 1 & 2 & 3 & 4 & 5 & 6 \\
\hline \multirow[t]{2}{*}{1} & a) Selectiva/Educación Inicial/Estudiantes jóvenes en su mayoría & $\mathrm{X}$ & $\mathrm{X}$ & $\mathrm{X}$ & & & \\
\hline & b) Abierta/Educación Permanente/Todas las edades & & & & $\mathrm{X}$ & $\mathrm{X}$ & $\mathrm{X}$ \\
\hline \multirow[t]{2}{*}{2} & a) Financiamiento público principalmente & $\mathrm{X}$ & & & & & - \\
\hline & b) Financiamientos de diversa índole & & $\mathrm{X}$ & & $\mathrm{X}$ & & - \\
\hline & c) Financiamiento privado predomina & & & $X$ & & $\mathrm{X}$ & - \\
\hline \multirow[t]{3}{*}{3} & a) Docencia \& Investigación ('+’: énfasis en investigación) & $\mathrm{x}$ & $X_{+}$ & & & & \\
\hline & b) Énfasis en Docencia & & & & $\mathrm{X}$ & $\mathrm{X}$ & \\
\hline & c) Especialización por misiones & & & $x$ & & & $\mathrm{x}$ \\
\hline \multirow[t]{2}{*}{4} & a) Enfocadas hacia lo nacional & $x$ & & & $\mathrm{x}$ & & \\
\hline & b) Dando importancia a la visión internacional & & $x$ & $X$ & & $X$ & $X$ \\
\hline \multirow[t]{2}{*}{5} & a) Status homogéneo de profesores e instituciones & $x$ & & & $\mathrm{x}$ & & - \\
\hline & b) Polarización en el status de profesores e instituciones & & $x$ & $\mathrm{x}$ & & $X$ & - \\
\hline \multirow[t]{2}{*}{6} & a) Bajo papel del e-learning y las TICs & $\mathrm{x}$ & & & & & \\
\hline & b) Alto papel del e-learning y las TICs & & $\mathrm{X}$ & $\mathrm{X}$ & $\mathrm{x}$ & $X$ & $\mathrm{x}$ \\
\hline
\end{tabular}

\section{Escenario 1: Tradición}

Las Universidades conservan su perfil actual. Siguen practicando simultáneamente la docencia y la investigación sin excesiva dependencia o involucramiento con el sector privado. Los Gobiernos de los países de la OECD siguen desempeñando un papel clave en el financiamiento de las universidades y en la regulación de su gestión. No se desarrollan muchas iniciativas dirigidas a obtener ganancias y/o financiamiento y la equidad, al igual que la rendición de cuentas, siguen siendo prioridades. La educación permanente y el e-learning se desarrollan ampliamente fuera del marco tradicional de las universidades.

\section{Escenario 2: Universidades Emprendedoras}

Instituciones selectivas que proveen de conocimientos a los jóvenes en su preparación inicial para la vida. La diferencia esencial con el escenario anterior es que las universidades (públicas o privadas) pueden actuar con gran autonomía ante una variedad de fuentes de financiamiento. Hay una 
mayor mezcla de los modelos de financiamiento público y privado. Los recursos para la universidad provienen de gran diversidad de fuentes. La investigación es considerada como una actividad importante y lucrativa. Sin embargo, las universidades toman esta actitud "orientada hacia el Mercado", sin perder los valores académicos básicos. Debido al prestigio e ingresos que recibe la investigación el profesorado mantiene un carácter elitista. La educación permanente se produce solo en universidades meramente docentes con un status más bajo que las universidades de investigación. Las tres misiones de la universidad - docencia, investigación y servicios a la comunidad (extensión) - están bien equilibradas, pese a la gran diferenciación entre IES. Se producen importantes acercamientos a los mercados internacionales y a las iniciativas de e-learning. Los recursos de las Universidades mejoran al igual que los salarios y el prestigio del profesorado.

\section{Escenario 3: Mercado Libre}

Las fuerzas del Mercado imperan en este escenario. Este sector privado está regulado por compañías privadas en lo que se refiere a la acreditación y garantía de la calidad y financiado a través de mecanismos de mercado. Las fuerzas del Mercado dan lugar a IES especializadas por función (docencia, investigación), área disciplinaria (administración de negocios, humanidades), tipos de audiencia (estudiantes jóvenes, a tiempo parcial, educación a distancia, educación de adultos, educación permanente), mientras que las corporaciones otorgan grados académicos a sus empleados mediante entrenamientos ad hoc. La jerarquía entre estas diversas IES se enfatiza mediante los ranking, aparece una súper-élite global y se produce una gran polarización en el status de los profesores. Se incrementa la competencia por captar estudiantes y los ingresos de matrícula. Estos últimos adquieren gran importancia como fuente principal de financiamiento de las IES. La Tecnología tiene una alta presencia en los métodos de enseñanza. La dimensión internacional del mercado educativo adquiere una relevancia alta. Debido a que la mayoría de los estudiantes y sus padres no están interesados en la investigación (que aumenta los costos de la matrícula), la investigación se traslada hacia centros de investigación públicos y hacia las divisiones de I \& D de las corporaciones. La investigación que permanece en las universidades adquiere un carácter aún más elitista, mientras que la docencia, en tanto que dirigida a mercados masivos, tiende a la estandarización del currículo y de los métodos docentes. La investigación tiende a responder a demandas específicas y suministra importantes ingresos vía los derechos de autor y propiedad 


\section{Escenario 4: Educación Permanente y Educación Abierta}

Las Universidades se caracterizan por el acceso universal -de acuerdo a la clasificación de Trow- para todas las edades y mucha menos investigación. La economía del conocimiento ha florecido y la educación superior es una fuente a la que se acude reiteradamente para lograr el desarrollo profesional financiada por compañías o por los propios individuos que aspiran a un ascenso social. En una sociedad que envejece, muchas personas cursan estudios universitarios como entretenimiento y no por razones profesionales. Las Universidades se orientan más hacia la demanda, los cursos cortos, la educación a distancia y el e-learning. Los Gobiernos o Agencias independientes tienen a su cargo la acreditación. La mayor parte de la investigación se realiza fuera del sistema de la educación superior. Los investigadores se trasladan de las universidades hacia las corporaciones, los institutos especializados, o hacia las pocas universidades de élite (World-Class Universities) que subsisten. Las Corporaciones y las universidades corporativas como Phoenix adquieren gran relevancia e influencia. La educación universitaria adopta un carácter profesionalizante lejano del modelo humboldtiano. La receptividad hacia las fuerzas del mercado es elevada en este escenario, en el que se produce una alta inversión en e-learning orientada a la ganancia.

\section{Escenario 5: Red Global de Instituciones}

Los estudios post-secundarios se ajustan a la demanda y a las necesidades del mercado. Dos innovaciones principales son: 1) Los estudiantes definen y diseñan sus propios cursos y grados académicos de entre los que se ofrecen. 2) Las IES forman partenariados con la industria. El e-learning tiene un gran desarrollo en este escenario. Los contenidos docentes tienden a la estandarización y modularización. La mayoría de la investigación se desarrolla fuera del ámbito del sistema de educación superior. Se produce una gran polarización del status académico, entre súper estrellas hábiles en el uso y desarrollo de "herramientas de aprendizaje" y profesores poco cualificados que imparten módulos estandarizados y que tienen un status mucho más bajo que los gurúes. Los programas y cursos adquieren una mayor relevancia que las instituciones. Los derechos de propiedad intelectual por aportes en la investigación y en los métodos de enseñanza dan grandes ingresos a sus propietarios.

\section{Escenario 6: Desaparición de las Universidades}

En este escenario el sector formal de educación superior terciaria desaparece. Las personas aprenden a lo largo de su vida - en el trabajo, en 
la casa - de acuerdo a motivaciones personales y profesionales, compartiendo experiencias en los mismos campos. Determinados conocimientos como la cirugía, por ejemplo, que requieren una cierta práctica se transmitirán a través de sistemas de aprendizajes ad hoc. La educación abierta será lo que predominará. El papel del aprendizaje mediante las redes tendrá un gran peso. Agencias especializadas acreditarán los conocimientos adquiridos por las personas mediante la presentación de credenciales específicas y pruebas a dichos efectos. La investigación tenderá a ser menos especializada en aquellas disciplinas que no requieren grandes inversiones para obtener resultados como las humanidades, mientras que la investigación que requiere grandes inversiones se llevará a cabo en instituciones públicas y en las divisiones de I \& D de las corporaciones.

El escenario 1 predomina en los países de Europa Occidental. En gran medida es el modelo por el que aboga la Declaración de la Conferencia Mundial de UNESCO sobre Educación Superior de UNESCO (1998).

Gráfico 1. Seis Posibles Escenarios para las Universidades

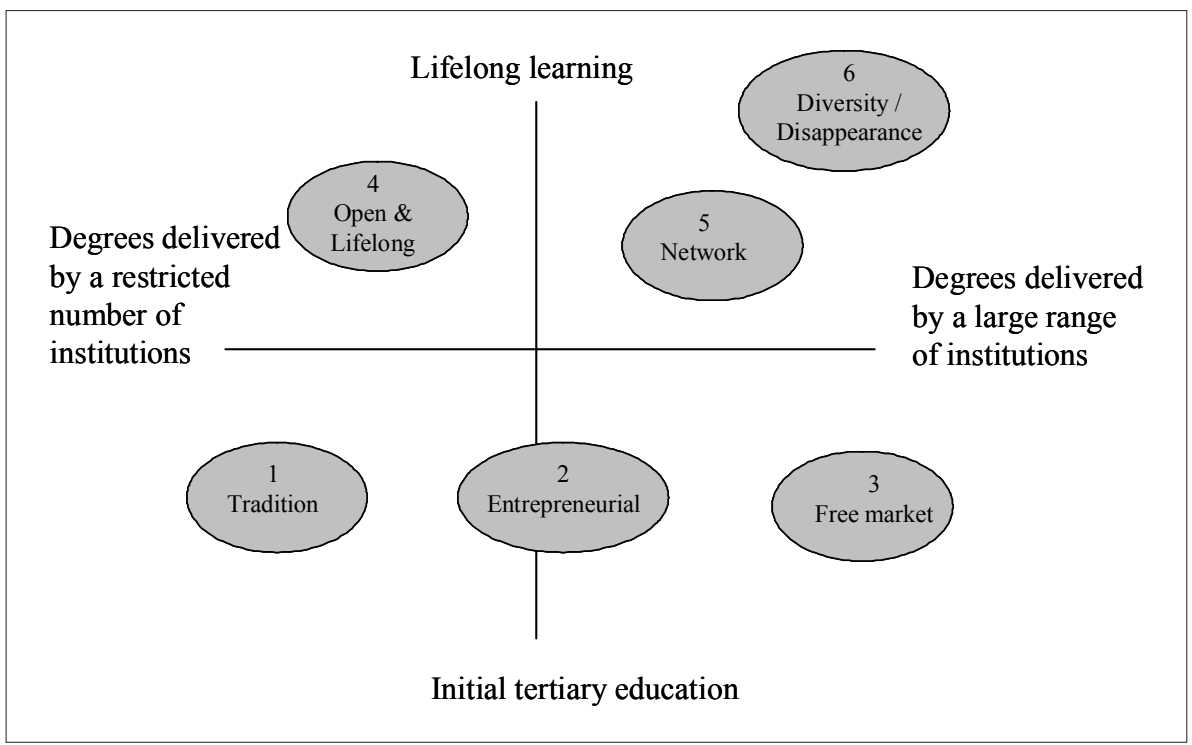

\section{Cuatro Escenarios de la OECD}

En un documento presentado en una reunión de Ministros de Educación, la OECD (junio, 2006) ha formulado cuatro escenarios para la 
educación superior que resumiremos a continuación, y que nos servirán de base para ciertas reflexiones y ejemplificaciones ${ }^{3}$.

Los objetivos son esclarecer el estado actual de la educación superior, qué modelo de esta debe impulsarse y como se logra esto.

\section{Escenario 1: Redes Abiertas}

La educación superior tiene un alto grado de internacionalización que implica intensos vínculos mediante redes diversas entre IES, profesores, estudiantes y actores diversos del sector productivo. Este modelo está basado más en la cooperación que en la competencia.

Se consolida el inglés como lingua-franca. Se mantiene una fuerte jerarquía entre las diversas IES. Las IES que no son propiamente universidades de investigación se benefician de los avances en el conocimiento en las universidades de ranking mundial. La Red brinda información en el estado del arte que puede ser usada libremente por académicos y estudiantes de diversos países. La cooperación voluntaria entre IES prevalece y se tiende a la armonización de los sistemas de educación superior. El proceso de Bologna es un modelo al respecto. ¿Será este modelo sustentable en una economía del conocimiento?

\section{Escenario 2: Sirviendo a las Comunidades Locales}

La educación superior es administrada y financiada por el Estado. Los académicos dirigen las IES y controlan los planes de estudio, programas, y otros aspectos claves de la enseñanza universitaria. Un pequeño número de IES de carácter elitario están vinculadas a redes internacionales. La IES promedio enfoca su docencia e investigación a los problemas nacionales y locales. Se enfatiza el estudio, a través de las ciencias sociales y las humanidades, de la cultura nacional y de los problemas sociales autóctonos.

\section{Escenario 3: Nuevo Tipo de Administración Pública}

Las IES privadas tienen su propio financiamiento. Las IES públicas dependen aún en gran medida de los fondos públicos. Sin embargo las IES tienden a diversificar sus fuentes de financiamiento y a depender menos de los fondos públicos. La venta de servicios diversos, patentes, consultorías

3 Vid www.oecd.org/edu/universityfutures 
y otras formas de diversificación implican una mayor autonomía financiera. Los estudiantes y sus familias pagan un porcentaje significativo del costo de sus estudios. Las fronteras entre la educación pública y la privada se hacen borrosas. Las IES son evaluadas y rinden cuentas periódicamente, tanto las públicas como las privadas, lo cuál contribuye a la obtención de fondos de fuentes diversas. Los préstamos estudiantiles se convierten en una forma importante del financiamiento de los estudios universitarios. El grueso de los proyectos de investigación son financiados de acuerdo a sus perspectivas económicas principalmente. Hay una fuerte competencia a nivel nacional entre las IES para obtener fondos que financien sus proyectos de investigación. La rendición de cuentas, la transparencia, la eficiencia y la efectividad están en el orden del día. ¿Podrá este modelo de educación superior dar respuestas más diversas y adecuadas?, o por el contrario tenderá a incrementar las desigualdades.

\section{Escenario 4: Educación Superior Inc}

En este escenario las IES compiten globalmente para proveer de servicios educativos y de investigación sobre bases comerciales. Se produce una desconexión entre docencia e investigación, como siempre lo han estado en el GATS. La universidad corporativa tipo Phoenix es el modelo que predomina. Las universidades son más bien seudouniversidades con programas específicos orientados a necesidades específicas del mercado. La educación superior se considera una mercancía y no un valor ético. Se produce una competencia fiera entre IES por estudiantes. Los servicios educativos y de investigación se incluyen en las negociaciones del GATS. Se refuerza la tendencia de algunos países - Australia, EE.UU., Malasia, Nueva Zelanda, Singapur, el Reino Unido..- de convertir su sector de educación superior en una industria de exportación. ¿Están los sistemas de educación superior de los países en desarrollo preparados para competir globalmente? ¿Qué ocurrirá con aquellas áreas del conocimiento enfermedades tropicales, contaminación ambiental - que no son comercialmente viables? ¿Qué impacto tendrá esto en las culturas y lenguajes nacionales? ¿Podrán sobrevivir?, o sucumbirán a una homogeneidad cultural y lingüística global?

El escenario 1 es el que está en vigor en Europa Occidental y constituye en cierta medida el ideal del Proceso de Bolonia. Aunque en este proceso hay fuertes tendencias a mezclar este escenario con aspectos del 3. El escenario 2 es propio de los sistemas de educación superior de países como 
Cuba y de algunos países latinoamericanos como Uruguay, donde aún la educación superior pública tiene un gran peso. El escenario 3 es típico de EE.UU. El escenario 4 está avanzando vertiginosamente desde los 90s, y está siendo impulsado por ciertos países miembros de la OMC que aspiran a convertir a la educación superior en un bien de importación y exportación, regulado por las normas de la OMC y conforme a las políticas del GATS.

\section{VISIÓN DE ESCENARIOS GLOBALES DE LA UNIVERSIDAD DESDE EL MUNDO ANGLOSAJÓN ${ }^{4}$}

1. Universidad Diversa: Especializadas en estudios multiculturales y en propuestas de transformación social.

2. Universidad Mundial: Dedicadas a problemas globales. En alguna medida equivalentes de las actuales World-Class y Research Universities.

3. Universidad Creativa: Priorizarán en vez de las profesiones tradicionales las nuevas: diseño, biotecnología, robótica, multimedia, ingenierías, ecología, desarrollo sostenible.

4. Universidad sin Divisiones artificiales: Concentrada en estudios transdisciplinarios.

5. Universidad del Ciberespacio: Operarán como Redes Virtuales.

6. Universidad de Adultos: Dedicadas a alumnos académica y emocionalmente maduros (por lo general de más de 30 años).

7. Universidad College: Formación de jóvenes egresados de los estudios secundarios. Además de formación académica se les ofrecerá cursos de formación personal.

8. Universidad a lo largo de la vida: Priorizarán el desarrollo de competencias dando menor importancia a los títulos formales.

9. Universidad Ubicua: Se vincularán a otros recursos formativos presentes en la sociedad: museos, hospitales, medios de comunicación, redes. Su objetivo será formar comunidades de aprendizaje con múltiples oportunidades de acceso y un alto grado de movilidad.

10. Universidad Laboratorio: Será un campo de experimentación para formas novedosas de educación superior.

4 James Duderstadt "Envisions the transformation to the 21st Century University. http://www.anc.org 


\section{VISIÓN DE ESCENARIOS GLOBALES Y REGIONALES DESDE AMÉRICA LATINA Y EL CARIBE}

Diversos especialistas de la región han formulado su visión de cuáles serían los escenarios globales ideales de la educación superior a nivel mundial pensando sobre todo en la región, otros han identificado las posibilidades de acuerdo a las diversas alternativas de política, y algunos han elaborado los escenarios pensando en el impacto de la globalización y de los acuerdos de libre comercio.

\section{1 ¿Competitividad privatizadora o cooperación solidaria?}

Sin una adecuada inversión en educación y, en especial, en educación superior y en las investigaciones universitarias, los países en desarrollo irán progresivamente a la bancarrota, las empresas perderán cada vez más su competitividad, los profesionales pasaran a ser recolectores de datos de los centros de investigación del Norte, en una sociedad del conocimiento en que los trabajadores del saber han ido sustituyendo progresivamente a los obreros industriales en el liderazgo, en dar carácter y perfil a la sociedad. El anterior contexto implica la emergencia de dos escenarios: uno, que profundiza en el tiempo la competitividad individualizada de las instituciones, de los académicos y de los estudiantes; y el otro, dirigido a una mayor cooperación horizontal entre instituciones y sectores, el cual se estructura en redes y en espacios comunitarios y trabaja en colaboración, sin por eso perder su identidad institucional. ${ }^{5}$

5 Los escenarios que predominen a nivel mundial influirán decisivamente con respecto a que escenario predominará en la educación superior en los diversos países. También los procesos nacionales serán decisivos al respecto. Visualizamos entre el 2007 y el 2030 tres escenarios posibles: 1) Hegemonía Imperial. 2) Ingobernabilidad Global. 3) Democracia Internacional Consensuada Hoy estamos viviendo en el primer escenario, que pudiera agudizarse en conflictividad si al concluir el mandato de Bush se elige un nuevo Presidente del Partido Republicano, lo que no parece probable. El escenario 2 podría ser el resultado de un cuestionamiento de la gobernabilidad imperial por potencias emergentes. Sería un escenario distinto al actual intento de "pax americana" y al escenario de mundo bipolar que prevaleció entre 1946 y 1989. La Ingobernabilidad Global se caracterizaría por un debilitamiento aún mayor de ONU y por el creciente conflicto entre los polos tradicionales de poder de la Alianza Atlántica y los nuevos poderes emergentes, por un lado, y por una fractura de la Unión Europea y EE.UU, por otro. El escenario 3 podría ser el resultado de la victoria de las negociaciones a nivel global y de transformaciones cualitativas esenciales en el sistema mundo capitalista actual. En este último escenario prevalecería a nivel mundial la visión de la educación superior como bien público. En los dos escenarios anteriores la visión de la educación superior como bien público seguiría teniendo vigencia en los países de Europa Occidental (y no de la Unión Europea en su totalidad), en algunos países asiáticos (China, Vietnam), y en aquellos países que tiendan a desprenderse en sus políticas de educación superior del "main stream" privatizador del sistema mundo, debido a procesos radicales de cambio social, tal y como ocurrió en Cuba en 1959 y está ocurriendo hoy en otros países de América Latina. En África seguiría teniendo mucha fuerza la visión de la educación superior como bien público. Por otra parte, en el Informe del Foro Mundial de Davos (2007) se presentan tres escenarios de riesgo global: 1) Pandemia: 
La alternativa 1 reforzaría el escenario de competitividad individualizada y mercantil. La alternativa 2 reforzaría el escenario solidario que considera la educación superior un bien público:

Alternativa 1. Se mantienen y se profundizan las medidas propuestas por los organismos internacionales del sector financiero, que corresponden a lo que se ha dado en llamar la política del Estado desertor.

Alternativa 2. Se otorga una elevada prioridad política a las áreas sociales del Estado, y entre ellas, en particular, a la educación, en todos sus niveles, y al desarrollo de la ciencia y a la tecnología. Es decir, se adoptan las principales recomendaciones de la Conferencia Mundial sobre la Educación Superior de la UNESCO. (Ver Tabla 2) (BROVETTO, 2000, p. 218).

\section{Tabla 2}

\section{TENDENCIAS ACTUALES EN LA PÓLEMICA SOBRE LA EDUCACIÓN SUPERIOR}

Alternativa No. 1

\begin{tabular}{|c|c|c|c|}
\hline & Financiamiento & $\begin{array}{l}\text { Se impulsa financiamiento } \\
\text { privado y se reduce el estatal }\end{array}$ & $\begin{array}{l}\text { El Estado asume su } \\
\text { responsabilidad financiera con } \\
\text { las IES públicas. Se } \\
\text { promueven fuentes } \\
\text { complementarias. }\end{array}$ \\
\hline 2. & $\begin{array}{l}\text { Participación del sector } \\
\text { privado }\end{array}$ & $\begin{array}{l}\text { Se promueve la creación de } \\
\text { IES privadas }\end{array}$ & $\begin{array}{l}\text { Se deja en libertad } \\
\text { controlando su calidad y } \\
\text { regulando su funcionamiento. }\end{array}$ \\
\hline & Acceso & $\begin{array}{l}\text { En respuesta a las demandas } \\
\text { del mercado. Regulado } \\
\text { (limitado) }\end{array}$ & $\begin{array}{l}\text { Se promueve y diversifica. Se } \\
\text { amplía la oferta pública }\end{array}$ \\
\hline 4. & $\begin{array}{l}\text { Estructura del sistema } \\
\text { de ES }\end{array}$ & $\begin{array}{l}\text { Se estratifica en diferentes } \\
\text { niveles por desagregación de } \\
\text { funciones. }\end{array}$ & $\begin{array}{l}\text { Se promueve la integración } \\
\text { entre los distintos niveles. }\end{array}$ \\
\hline 5. & $\begin{array}{l}\text { Creación del } \\
\text { conocimiento }\end{array}$ & $\begin{array}{l}\text { Con fin económico } \\
\text { incorporado al capital. }\end{array}$ & $\begin{array}{l}\text { Con fin social incorporado al } \\
\text { desarrollo humano. }\end{array}$ \\
\hline 6. & $\begin{array}{l}\text { Distribución del } \\
\text { conocimiento }\end{array}$ & Restringida & $\begin{array}{l}\text { Amplia, generadora de } \\
\text { demandas sociales. }\end{array}$ \\
\hline & Gobierno & Manejo desde la Gerencia & $\begin{array}{l}\text { Participación responsable de } \\
\text { todos los estamentos. }\end{array}$ \\
\hline
\end{tabular}

Fuente: Brovetto, J., (2000, p. 229).

una pandemia con un virus que da lugar a miles de muertos y revueltas que alteran la geopolítica global. 2) Cambio Climático: las catástrofes que origina el cambio climático dan lugar a una toma de conciencia y un afán de corregir las desigualdades económicas globales. 3) "Shock" Petrolero: se dispara el precio del petróleo lo que implica tensiones de envergadura en la geopolítica global, recesión económica y no priorizar la lucha contra el cambio climático. 


\section{2 ¿Bien público o bien de consumo privado?}

Se han dado cuatro tipos de respuestas (a la manera de escenarios) al dilema de sí la educación superior debe considerarse un bien público o bien un servicio de consumo privado:

- La educación superior es un bien público. En este caso se justifica la preponderancia de la intervención del Estado. Puede llegar a una forma extrema de monopolio estatal con la supresión o gran limitación de la educación superior privada.

- La educación es un bien privado. Esto implica que la intervención del Estado no está justificada, debiendo la oferta educativa estar asegurada mediante un mercado libre y desregulado.

- La educación superior es un bien predominantemente público que produce beneficios privados. El Estado, por tanto, debe asumir gran parte del financiamiento, aunque compartiendo esta carga económica con los alumnos, sus familias y los futuros empleadores.

- La educación superior es un bien esencialmente privado que produce beneficio público. Esto implica que el Estado contribuya significativamente al financiamiento de los servicios educativos, pero reduciendo su intervención al mínimo para permitir el funcionamiento de un "casi-mercado educativo", sustentado en la concurrencia y autonomía que prestan este servicio (DIAS SOBRINHO, 2005, p. 137).

\section{3 ¿Subordinación a la Globalización o Cooperación Independiente?}

Partiendo de la identificación de dos dinámicas - la del GATS y la de la visión de la educación superior dirigida a obtener ganancias; y de la crítica y el rechazo a considerar la educación superior una mercancía y no un bien público- pudieran formularse cinco escenarios para la educación superior latinoamericana (GARCIA GUADILLA, 2002; LÓPES SEGRERA, 2006).

Escenario A: Globalización con subordinación y mercado actual "indefinido"

Este escenario sería la continuación del escenario que existe actualmente en América Latina. La tendencia es a la privatización creciente de la educación superior. 
Escenario B: Globalización con interacción y mercado regulado por acuerdos educativos

Este escenario implicaría el predominio de lo público sin excluir lo privado, con una visión de la educación superior como un bien público, creadora de valores ciudadanos. Los Acuerdos del Grupo Montevideo parecen avanzar en este sentido. Pese a las críticas, muchos consideran que el Espacio Europeo de Educación Superior (EEES) se regiría por esta visión. UNESCO, pese a ciertos cambios, y a la designación ambigua de la educación superior como un "bien público global" en algunos documentos, también parece seguirse moviendo en esta dirección.

Escenario C: Globalización con subordinación y mercado regulado por acuerdos comerciales (como GATS)

Implicaría subordinarse a las políticas del GATS, que ha planteado incluir la educación superior como un bien de importación y exportación, regulado por sus normas. Esto sería funesto para los países en vías de desarrollo, pues implicaría una desnacionalización de su educación superior ante la llegada indiscriminada de nuevos proveedores con ánimo de lucro.

Escenario D: Globalización con interacción y mercado regulado por acuerdos comerciales (como el GATS)

"Los países estudian y conocen el posible impacto de los acuerdos comerciales. Manejan adecuadamente las desventajas, y tratan de sacar el máximo provecho de las ventajas.

Escenario E: Globalización sin subordinación y con cooperación independiente

Un creciente número de países - Cuba, Venezuela, Bolivia, Ecuador, Uruguay...- establecen acuerdos de cooperación en el ámbito universitario sin subordinarse a los acuerdos comerciales del GATS.

\section{ESCENARIOS GLOBALES Y REGIONALES: ASIA, ÁFRICA Y LOS ESTADOS ÁRABES}

Corriendo el riesgo de simplificar un problema complejo y en permanente evolución, podríamos decir que mientras en Europa Occidental y en África prevalece el modelo de educación superior financiado por el Estado, en Asia, América Latina y el Caribe y los Estados Árabes, la educación superior privada avanza aceleradamente como respuesta a la masificación. 
En el caso de África pudieran enunciarse dos escenarios: uno de creciente "fuga de cerebros" y otro de construcción de capacidades que permitan a la región proporcionar condiciones adecuadas de trabajo a sus profesionales para evitar su emigración masiva. Esto implica alternativas de política diversas a las que predominan hoy, no solo por parte de los países africanos, sino también de los países desarrollados.

Asia es un mosaico de países de naturaleza muy diversa. Esto hace difícil formular escenarios que abarquen toda la región. Un escenario pudiera ser el de Globalización con hegemonía. Esto implicaría que los países más desarrollados - Japón, Corea del Sur, Singapur- y/o poblados -China, India - de la región, tuvieran un gran impacto en los sistemas de educación superior de los menos desarrollados a través de nuevos proveedores con ánimo de lucro. Otro escenario podría ser el de Privatización ante la Masificación. En grandes países como Japón, Corea del Sur, Taiwán, Filipinas e Indonesia, los estudiantes en su mayoría - en algunos casos en un $80 \%$ - están matriculados en universidades privadas. Aún en zonas de Asia como China, Vietnam y Asia Central, donde la educación privada apenas existía o no existía en absoluto, observamos el veloz crecimiento de la educación superior privada.

También en los Estados Árabes el escenario de la privatización acelerada parece predominar, junto a la tendencia - que tiene gran importancia en Asia - de enviar a estudiar al extranjero un alto número de estudiantes.

\section{CONCLUSIONES}

El escenario 1 del epígrafe 1 es él que está en vigor en los países de Europa Occidental y es el ideal de muchos países en desarrollo. En la Declaración de la Conferencia Mundial de UNESCO sobre Educación Superior de UNESCO (1998), este modelo es en gran medida considerado un ideal, o al menos una opción mejor que las tendencias privatizadoras que auspicia el Banco Mundial. En la Tabla 2 del actual Ministro de Educación de Uruguay, Jorge Brovetto, también este es el modelo por el cuál se apuesta. Este escenario se corresponde con el 1 y el 3 del epígrafe 4.2 Bien Público o bien de consumo privado y con el B -globalización con interacción y mercado regulado por acuerdos educativos- y el E globalización sin subordinación y con cooperación independiente -de los escenarios formulados para América Latina en el epígrafe 4.3. También se correspondería con el escenario de construcción de capacidades en África.

Estas reflexiones nos llevan a la siguiente consideración: ¿Cuál de estos escenarios consideramos el ideal a nivel mundial? ¿Por qué razones? 
¿Qué alternativas de políticas debemos apoyar para impulsar el mejor escenario posible a nivel nacional, regional y global? ¿Acaso no es necesario construir un nuevo escenario más deseable que los que existen? También podríamos plantearnos lo siguiente: ¿no será que en el futuro coexistirán escenarios diversos de carácter heterogéneo de acuerdo a las características diversas de naciones y regiones? ¿Hay alguna correlación a nivel de país entre IDH, predominio de la educación superior pública y políticas para lograr un desarrollo sostenible? ¿Qué alternativas de políticas de educación superior mejorarían el IDH y el desarrollo sostenible a nivel de país, región y mundialmente? ¿Con qué políticas podremos construir los mejores escenarios? ¿Cuales nos conducirán a los peores escenarios? La situación que prevalezca en el ámbito geopolítico, como ya hemos señalado, será esencial al respecto.

Para elaborar escenarios es clave que variables seleccionemos para construirlos. Una vez elaborados tendremos una guía para formular aquellas políticas educacionales (alternativas) que tenderán a fortalecer lo que consideramos el mejor escenario posible. La OCDE, por ejemplo, privilegia en sus ejercicios de prospectiva variables tales como: 1) Tipo de población con cobertura y por ende acceso a la educación terciaria. 2) Naturaleza del financiamiento (público, privado, mixto). 3) Dimensión internacional del sistema de educación superior. 4) Homogeneidad y heterogeneidad del status de las IES y del profesorado. 5) Grado de e-learning en las IES y de equipamiento en TICs.

En los países en desarrollo es clave adecuar el conocimiento relevante de carácter global a una agenda local que establezca prioridades. Más importante que dedicar recursos a obtener Nobels, es crear capacidades que produzcan investigaciones relevantes socialmente y que permitan construir recursos humanos y sociales. Estas prioridades deben reflejarse en las metodologías de construcción de escenarios.

\section{REFERÊNCIAS}

BROVETTO, J. La educación superior para el siglo XXI. En: TÜNNERMANN, C.; LÓPEZ SEGRERA, F. La educación en el horizonte del siglo XXI. Caracas: IESALC/UNESCO, n. 12, 2000. Colección Respuestas.

DIAS SOBRINHO, J.. Dilemas da educação superior no mundo globalizado, São Paulo: Casa do Psicólogo, 2005. 
GARCÍA GUADILLA, C. ACGS (GATS) Educación superior y América Latina. Disponível en: www.google.es/search?q=escenarios. Acesso en: 2002.

LANCRIN, S. V. Building future scenarios for universities and higher education. In: POLICY futures in education. Acesso en: 2004. Disponível en: <www.wwwords.co.uk/PFIE>

LÓPEZ SEGRERA, F. Escenarios mundiales de la educación superior. Buenos Aires: CLACSO, 2006. 